\title{
Stretch-induced functional disorder of axonal transport in the cultured rat cortex neuron
}

\author{
Shigeru Aomura ${ }^{1}$, Hiromichi Nakadate ${ }^{1 *}$, Yuma Kaneko ${ }^{1}$, Akiyoshi Nishimura $^{2}$ and Remy Willinger $^{3}$ \\ ${ }^{1}$ Graduate School of System Design, Tokyo Metropolitan University, Tokyo, Japan \\ ${ }^{2}$ Institute of Biomedical Sciences, Tokushima University, Tokushima, Japan \\ ${ }^{3}$ Université de Strasbourg ICube, UNISTRA-CNRS, Strasbourg, France
}

\begin{abstract}
Background: Diffuse axonal injury (DAI) is a one of the main causes of traumatic brain injury and caused by the impulsive stretching of neuronal axons resulting from rapid deformation of a brain. DAI is characterized by a gradual swelling of the axons which is formed by the accumulation of cellular organelles and proteins, and the swelling is the morphological hallmark of DAI pathology. In recent years, the details of the stress and strain of the whole damaged brain are becoming clear with the rapid development of the computational mechanics and finite element (FE) head models are able to give better prediction to the brain injury and to evaluate the protective safety methods with more detailed neuronal tolerance criteria. In this study, axonal injury induced by precisely controlled impulsive strain and strain rate was evaluated and the tolerance criteria for the functional disorder for each dysfunction and disruption level of axonal transport was obtained by observation of $\beta$-amyloid precursor protein $(\beta-\mathrm{APP})$ in cultured rat cortex neuron.

Methods: The uniaxial stretching device which could give various combinations of strains and strain rates to neurons was developed. The various loading conditions for neurons were verified by comparing the experimental displacement history of the substrate taken by microscopy with the FE strain distribution analysis of culturing substrate. The primary rat cortex neurons were stretched by different combinations of strains and strain rates and $\beta$-APP was immunostained at $3 \mathrm{~h}$ after loading and observed by fluorescence microscopy.

Results: The number of swellings and bulbs formed on axons by $\beta$-APP-accumulation after stretching were observed and counted by fluorescent images. The dysfunction of the axonal transport was defined as the rate of neurons that have $\beta$-APP-accumulating axonal swellings and disruption of the axonal transport was defined as the rate of neurons that have $\beta$-APP-accumulating axonal bulbs, respectively. The degree of the functional disorder of the axonal transport advanced with the increase of strain and strain rate.

Conclusions: The mechanical threshold of dysfunction and disruption of axonal transport were the strain with 0.22 and the strain rate with 27 /s. The intervals between swellings on an axon are constant and do not depend on the axonal injury level nor the magnitude of the strain of the axons.
\end{abstract}

\section{Background}

Diffuse axonal injury (DAI), which involves widespread damage to neural cells, is caused by sudden inertial loading to the head, and is accompanied with the stretching of neural axons resulting from the rapid deformation of brain tissue [1]. This mechanical insult damages the neurofilament structure in the axonal cytoskeleton, causing focal compaction and/or impaired transport. The resulting accumulation of neurofilaments and transport materials induces focal axonal swelling [2]. Axonal swelling leads to secondary axotomy, resulting in the disconnection of neurons from target tissues and cell death $[3,4]$.

The axonal swelling associated with the accumulation of cellular organelles and proteins is the morphological hallmark of DAI pathology [5]. In the past, histological diagnosis of DAI was made by silver staining for the presence of, previously referred to as, "axonal retraction ball", however, it generally takes $12-24 \mathrm{~h}$ before retraction ball appears after head injury [6]. Earlier detection can be achieved by immunolabeling the $\beta$-amyloid precursor protein ( $\beta$-APP) which is conveyed by axonal transport [7]. $\beta$-APP accumulates where axonal transport is disrupted [8] and the time to a detectable level of $\beta$-APP is as early as $1.75-3 \mathrm{~h}$ after head injury [9-11]. Wilkinson et al. [12] investigated the relationship between the size of the swellings of the damaged axon and survival time in postmortem brain tissue by measuring the minimum diameter of the $\beta$-APP-immunolabelled damaged axons and reported that there was a strong, positive and significant relationship between the mean size of axonal swellings and survival time.

The better understanding of the relationship between the biomechanics and the pathophysiology of various phases of axonal trauma may give future advancements in the prevention and treatment of traumatic axonal injury. Recently, finite element (FE) models of human head including the detailed brain structure have been developed as one of the biomechanical approaches and are considered to be the most powerful tool to investigate the response of the human head to the various impact conditions. FE models are not only able to predict stress and strain of the brain under various impact conditions but also have the capability to predict injury and death of cells with more detailed tolerance criteria of nerve cells. Tolerance criteria dependent on tissue level measures of loading should be provided with more compatible form to the FE models from a mechanical point of view.

Correspondence to: Hiromichi Nakadate, Graduate School of System Design, Tokyo Metropolitan University, Tokyo, Japan, E-mail: nakadate@tmu.ac.jp

Key words: traumatic brain injury, diffuse axonal injury, $\beta$-amyloid precursor protein, axonal swelling, axonal bulb, injury threshold, axonal transport, finite element method, axonal stretching, impulsive strain

Received: May 02, 2016; Accepted: May 16, 2016; Published: May 18, 2016 
Since the brain is a viscoelastic biological tissue, its mechanical response is dependent on both the magnitude and the rate of strain. When axons are exposed to impulsive stretching, strains are always accompanied with various strain rates. The axonal injury resulting from brain tissue deformation should be investigated with the dynamically controlled strain related with strain rate, where the time history of the displacement of the substrate must be generated repeatedly and stably. However tolerance criteria for strain and strain rate compatible with FE models are difficult to obtain by in vivo models because of a lack of an accessibility to the tissue [13].

However the swellings along the axons observed at the early stage of axonal injury can be reproduced by tensile and shear stress on in vitro models and the pathology has a remarkably similar appearance of axonal swellings found after diffuse brain injury in humans suffering brain trauma [14-17]. Pfister et al. [14] realized an in vitro uniaxial stretch model for axonal injury, where strain and strain rate were independently controlled and the effect of the strain rate on neural stretch injury was observed. However the difference between dysfunction and disruption of the axonal transport is not observed and the quantitative evaluation of dysfunction and disruption is not mentioned. The quantitative relation between impulsive strain and axonal injury in which the axonal swellings and the bulbs are observed independently is still unclear.

In this study, axonal injury induced by impulsive stretching is evaluated by observation of $\beta$-APP in cultured rat cortex neuron. Dysfunction of the axonal transport and disruption of the axonal transport were independently defined and observed and the mechanical threshold of dysfunction and disruption of axonal transport are evaluated.

\section{Methods}

\section{Uniaxial stretching device and PDMS chamber}

Under the dynamic loading condition, the results of uniaxial stretching is more appropriate than biaxial deformation on a membrane in order to quantitatively appreciate the influence of strain rate on neuronal damage by stretching. A uniaxial stretching device was developed so as to realize the uniaxial strain with various strain rates. The polydimethylsiloxane (PDMS) chamber with a thin substrate was manufactured. The strain distribution around the center of the substrate on which neurons were cultured was calculated numerically by finite element method (FEM).

The results showed the ratio of the strain perpendicular to the strain in the stretching direction stays within $10 \%$. The uniaxial stretching device consists of a servo actuator (RCS3-SA8C, IAI, Shizuoka, Japan), a servo actuator controller (SCON-C; IAI), a linear sensor for measuring tensile displacement (LP-20F, Midori Precisions, Tokyo, Japan), a load cell for measuring tensile loading (TCLS, Toyo Sokki, Kanagawa, Japan), a load cell converter (LC14111, Unipulse, Tokyo, Japan), a programmable logic controller, an A/D converter unit (KV-3000 CPU, Keyence, Osaka, Japan), an AC power unit (KV-U7, Keyence) and a polydimethylsiloxane (PDMS) chamber, and is shown in Figure 1(A).

The PDMS chamber is clamped to the microscope stage at one edge and the other edge of the chamber is connected to the stainless plate, and the displacement of the stainless plate is stopped by hitting the stopper (B1). The stainless plate is connected to the slider of the actuator through a wire and other tip of the wire is connected to an
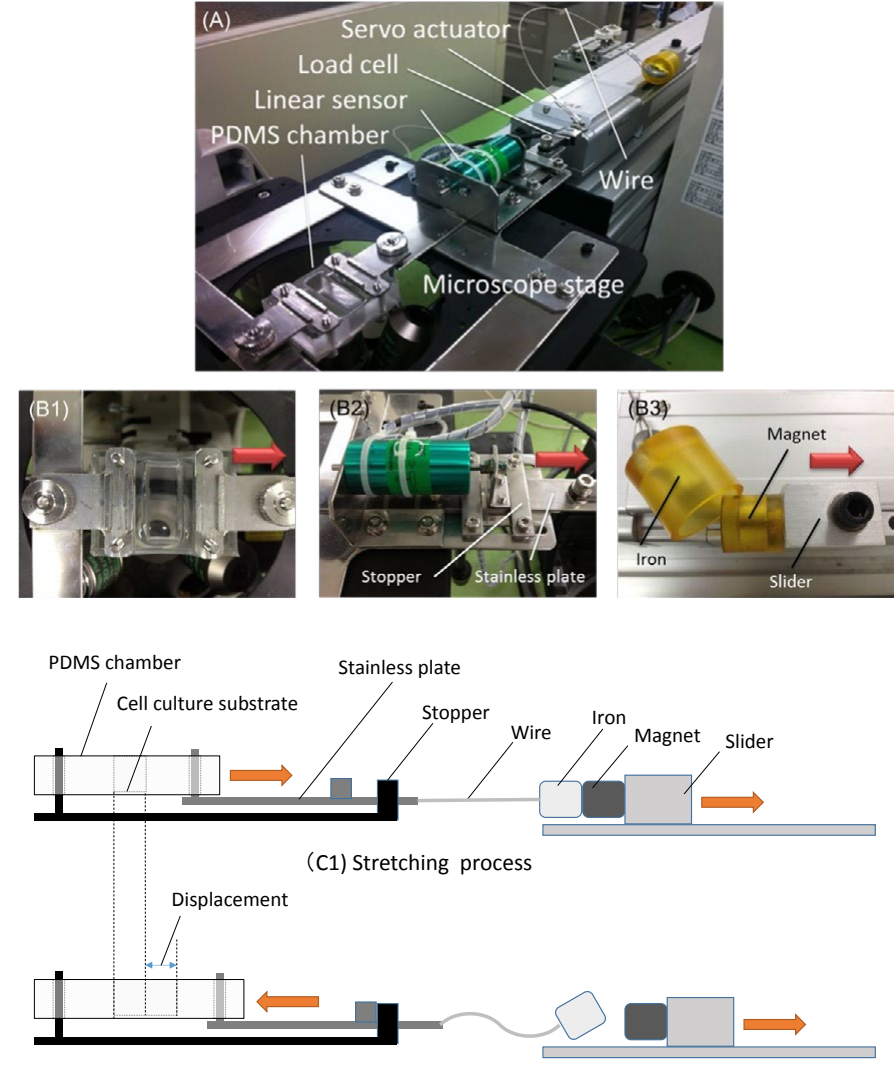

(C2) Releasing process

Figure 1. Uniaxial stretching device

The uniaxial stretching device and its components. The device consists of a PDMS chamber on a microscope stage, a linear sensor, a load cell, a servo actuator and a wire (A). The PDMS chamber is clamped to the microscope stage at one edge, and the other edge of the chamber is connected to the stainless plate those displacement is stopped by hitting the stopper (B1). The stainless plate is connected to the slider of the actuator through a wire and other tip of the wire is connected to an iron piece (B2). The iron piece is attracted strongly with the magnet set on the slider. (B3). The schematic illustration of the deviceloading mechanism is shown for the stretching process $(\mathrm{C} 1)$ and the releasing process $(\mathrm{C} 2)$, respectively.

iron piece (B2). The iron piece is attracted strongly with the magnet set on the slider (B3). The schematic illustration of the device-loading mechanism is shown in Figure 1, the stretching process $(\mathrm{C} 1)$ and the releasing process (C2), respectively, and both the stretching and the releasing process are described as follows, respectively. The stainless plate connected to the PDMS chamber is pulled by the servo actuator through the wire in the direction of the arrow as shown in $(\mathrm{C} 1)$. The stainless plate is pulled and moved until the plate hits the stopper. The stainless plate stays at original position during acceleration of the actuator by the slack of the wire and the chamber is pulled suddenly at a constant velocity once tension is applied to the wire. The substrate fixed at the bottom of the PDMS chamber is stretched at the same time as the PDMS chamber is stretched. On the last stage of the stretching process, the iron piece is detached from the magnet on the slider by the stainless plate hitting the stopper and stopping suddenly (C2). Subsequently, the PDMS chamber and substrate stretched return to the original state by elastic force. Through the process mentioned above, the device can generate the semi-sinusoidal displacement waveform during stretching and releasing.

The basic structure of the PDMS chamber with a well and a thin substrate is shown in Figure 2(A). The base, a PDMS-prepolymer and 


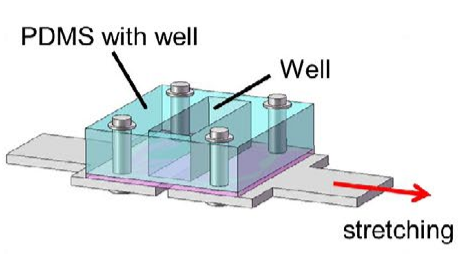

(A)

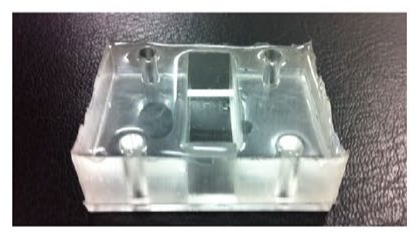

(C)

Figure 2. PDMS chamber

PDMS chamber with a well fixed on a stainless steel plate at four points, the thin PDMS substrate with $0.3 \mathrm{~mm}$ thickness is glued and baked under the PDMS plate with $10 \mathrm{~mm}$ height. The chamber is stretched by the stainless steel plate fixed under the chamber (A), the cells are cultured on the center area of the bottom surface of the well with $10 \mathrm{~mm} \times 30$ $\mathrm{mm}(\mathrm{B})$. PDMS chamber fired and hardened (C).

the curing agent (SYLGARD 184 Silicone Elastomer Kit, Dow Corning, Midland, MI, USA) were mixed in 10:1 mass ratio and the mixture was deaerated in a round desiccator connected to a dry vacuum pump. The deaerated mixture was poured into a polystyrene square case and stainless mold to be 0.3 and $10 \mathrm{~mm}$ in thickness (B), respectively, and was cured on a hot plate at $65^{\circ} \mathrm{C}$ for $1 \mathrm{~h}$. After curing, the two parts were bonded by the deaerated mixture and cured at $65^{\circ} \mathrm{C}$ for $1 \mathrm{~h}(\mathrm{C})$.

\section{Strain distribution analysis of culturing surface by FEM}

The numerical analysis of the strain distribution of the culturing surface was carried out by FEM. The FE model of the chamber is shown in Figure 3(A). A direction of the $\mathrm{x}$ axis corresponds to the stretching direction and the $\mathrm{y}$ axis corresponds to the perpendicular to the stretching direction in the coordinate system.

Numerical calculation was carried out by FEM code LS-DYNA ver.971 by LSTC (Livermore Software Technology Corporation, CA, USA) and the Jvision 3.0 by JSOL (Tokyo, Japan) was used as the preand post-processor. The material property of the chamber model is shown as follows [18]. The density: $9.2 \times 10^{2} \mathrm{~kg} / \mathrm{m}^{3}$, Young's modulus: $0.75 \mathrm{MPa}$, Poisson's ratio: 0.49

The results of numerical analysis for the stretching are shown in Figure 3(B) and (C). The chamber was stretched $4 \mathrm{~mm}$ with the velocity $0.5 \mathrm{~m} / \mathrm{s}$. The strain distribution of the whole culturing surface in longitudinal direction, i.e. the direction of the $\mathrm{x}$ axis is shown in Figure 3(B1) and the strain distribution in perpendicular to the stretching direction, i.e. the direction of the y axis is shown in Figure 3(B2). The strain for stretching direction (B1) shows tension and the strain for perpendicular to the stretching direction (B2) shows compression. The strain by stretching was evaluated on the four elements of the culturing surface. The four points a, b, c, and d are shown in Figure 3(C1). The strains in the direction of $\mathrm{x}$ axis on four points are shown in Figure $3(\mathrm{C} 2)$ and those in the direction of $y$ axis are shown in Figure $3(\mathrm{C} 3)$, respectively. The maximum tensile displacement of $4 \mathrm{~mm}$ caused the tensile strain of 0.3 in the stretching direction on point $b$, point $c$ and point $\mathrm{d}$, and the tensile strain of 0.25 was caused on the point which locates rather close to the end of the culturing surface (shorter edge) as shown in Figure $3(\mathrm{C} 2)$. And the compressive strain $0.02-0.03$ perpendicular to the stretching direction was caused around the center of the culturing surface on point $c$ and point $d$, and the strain increased as closing to the edge and the rather bigger compressive strain of $0.06-$ 0.07 was caused on the point a as shown in Figure 3(C3). The tensile strain with 0.3 was obtained on the point $c$ in the stretching direction and compressive strain in the perpendicular direction 0.02-0.03.

The results of numerical calculation show that the compressive strain in perpendicular direction can be controlled smaller than $10 \%$ compared with the tensile strain in the stretching direction.

\section{The displacement history of the center of the culturing surface}

The deformation of the center of the culturing surface in the PDMS chamber was measured experimentally. The PDMS chamber was pulled $4 \mathrm{~mm}$ in total by $0.5 \mathrm{~mm}$ every time by the uniaxial stretching device. The center area of the culturing surface was observed and the photos were taken by microscope, and the strain around the center was calculated by image analysis based on the mark attached on the surface.

The Green-Lagrange strains of the culture substrate in the PDMS chamber were calculated by microscope images before and after the stretching. The strains on the central point of the culturing substrate in the stretching direction and perpendicular direction were obtained for every $0.5 \mathrm{~mm}$ displacement to $4 \mathrm{~mm}$ in total and plotted in Figure 4(A). In the experiment, the compressive strain perpendicular to the stretching direction was smaller than 0.03 for the tensile strain 0.3 in the stretching direction at the maximum displacement with $4 \mathrm{~mm}$. The history of the displacement of the central point for various stretching speeds are shown in Figure 4(B). The impact 1 corresponds to the strain of 0.10 at the strain rate $11 \mathrm{~s}^{-1}$, the impact 2 corresponds to the strain of 0.15 at the strain rate $21 \mathrm{~s}^{-1}$, the impact 3 corresponds to the strain of 0.22 at the strain rate $27 \mathrm{~s}^{-1}$ and the impact 4 corresponds to the strain of 0.30 at the strain rate $38 \mathrm{~s}^{-1}$, respectively. The curves stood up in the first $10 \mathrm{msec}$ by stretching, and converged in the last $10 \mathrm{msec}$ by releasing with the same ratio as the standing up. It is expected that the neuron on the substrate is stretched/compressed with a similar deformation history through the substrate.

\section{Culturing the primary rat cortex neurons}

Primary rat cortex neurons (Gibco, Life Technologies, Carlsbad, CA, USA) were cultured in Neurobasal medium (Gibco) supplemented with $2.5 \mathrm{~mL} / \mathrm{L}$ GLUTAMAX-I (Gibco) and $20 \mathrm{~mL} / \mathrm{L}$ B27 Supplement (Gibco) under conditions of $5 \% \mathrm{CO}_{2}$ and $100 \%$ humidity at $37^{\circ} \mathrm{C}$. The cells were seeded into Poly-D-lysine-coated culture substrate $(10 \mathrm{~mm} \times$ $30 \mathrm{~mm}$ ) of PDMS chamber at $6 \times 10^{4}$ cells $/ \mathrm{cm}^{2}$ for $7-9$ days as shown in Figure 5. The neurons matched in age to those subjected to strains were cultured in the PDMS chamber as a sham control, after which they were set in and removed from the uniaxial stretching device without receiving any mechanical load.

The medium in the PDMS chamber was not removed during the experiment which was completed within $5 \mathrm{~min}$. The temperature of the device and surroundings were kept at $37^{\circ} \mathrm{C}$. The PDMS chamber was returned to the $\mathrm{CO}_{2}$ incubator after the experiment.

\section{Uniaxial stretching for axonal injury}

A primary effect of dynamic deformation of axons following 


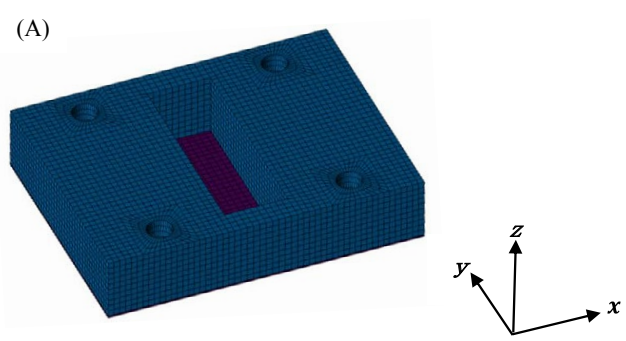

(C1)

(B1)

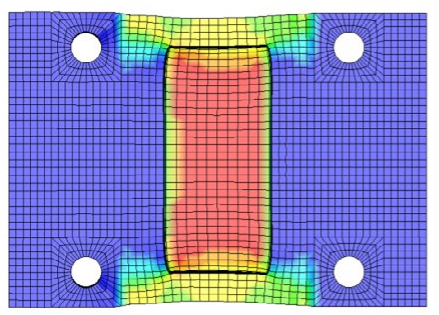

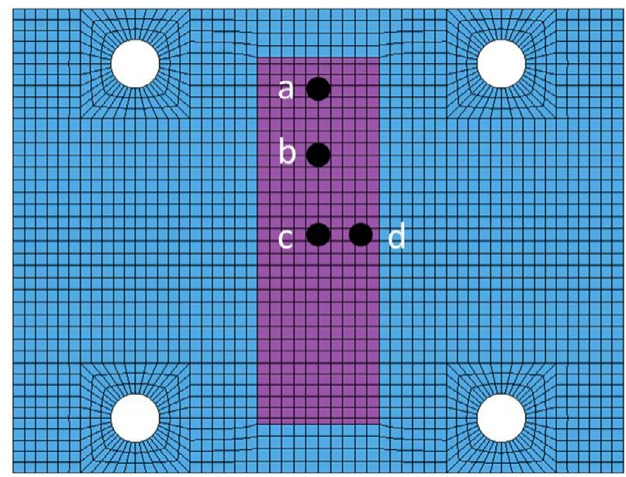

[strain]

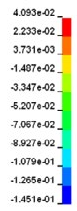

$(\mathrm{C} 2)$

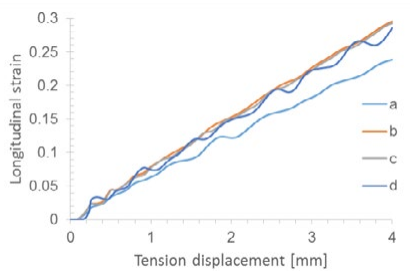

(C3)

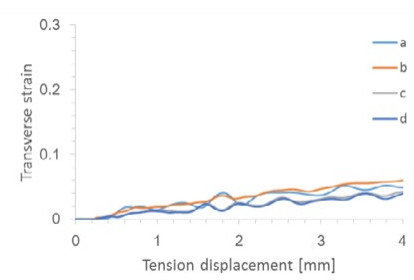

Figure 3. Strain distribution analysis of PDMS chamber by FEM

The strain distribution of the PDMS chamber was obtained by finite element analysis. The finite element model of the PDMS chamber (A). The strain distribution of longitudinal direction (B1) and the transverse direction (B2) of the culture substrate at $4 \mathrm{~mm}$ displacement of the PDMS chamber. Relation between the tensile displacement and the longitudinal strain (C2) or transverse strain $(\mathrm{C} 3)$ at four points $(\mathrm{C} 1)$ of the culture substrate are shown.

stretching is the disruption of axonal transport, resulting in accumulation of transported materials in axonal swellings within just hours [17]. Swellings appear in a periodic interval along the connected axons, like beads on a string, to form a pathological phenotype referred to as "axonal varicosities" [19]. Axonal pathology found shortly after traumatic brain injury (TBI) is a single swelling at a disconnection point on an axon, described as a terminal "axonal bulb" (previously referred to as a "retraction ball") $[1,19-21]$. In this study, $\beta$-APP was stained at $3 \mathrm{~h}$ post loading and observed by using an inverted fluorescence microscopy (IX71, Olympus, Japan) equipped with a fluorescence mirror unit (U-MWIG3, Olympus). Cultures were rinsed with Dulbecco's phosphate-buffered saline (DPBS) with $\mathrm{Ca}^{2+}$ and $\mathrm{Mg}^{2+}$ and fixed with $4 \%$ paraformaldehyde for 20 minutes at room temperature.

After permeabilization with $0.3 \%$ Triton X-100 for 5 minutes at room temperature, cultures were blocked with $5 \%$ goat serum for 60 minutes at room temperature and incubated in rabbit polyclonal anti-
$\beta$-APP (51-2700, Invitrogen, Life Technologies) at a dilution of 1:50 as the primary antibody overnight at $4^{\circ} \mathrm{C}$. Subsequently, $10 \mu \mathrm{g} / \mathrm{mL}$ Alexa Fluor 594-conjugated goat anti-rabbit $\operatorname{IgG}(\mathrm{H}+\mathrm{L})$ secondary antibody (A-11037, Molecular Probes, Life Technologies) was applied for 60 minutes at room temperature. B-APP-accumulated axonal swellings and axonal bulbs formed after stretching were observed and counted quantitatively by fluorescent images as shown in Figure 6 .

Dysfunction of the axonal transport was defined as the rate of neurons that have $\beta$ - APP accumulated axonal swellings. Disruption of the axonal transport was defined as the rate of neurons that have $\beta$-APP-accumulated axonal bulb. In addition, we evaluated the number of axonal swellings per $100 \mu \mathrm{m}$ and the length of the axon in which axonal bulb was observed, respectively. The axon length was measured manually by Image J (National Institutes of Health, Bethesda, MD, USA). Results were expressed as the mean \pm standard deviation (SD) of 4 independent experiments. 200-300 neurons per a PDMS substrate 
(A)

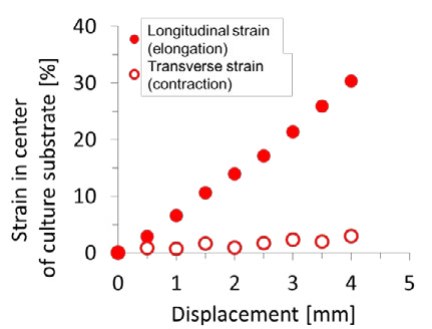

(B)

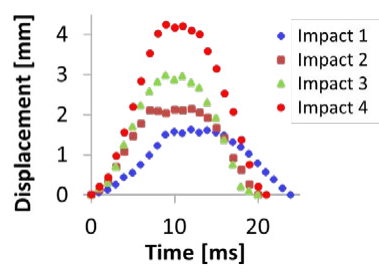

Figure 4. Displacement measurement of PDMS chamber

The strain and displacement of the culturing substrate fixed on the PDMS chamber for the uniaxial stretching were observed experimentally. Relation between the forced displacement and distortions around the center where cells are cultured was obtained. The strain in the direction perpendicular to the stretching direction remains within $10 \%$ compared with the longitudinal strain (A). The displacement corresponds to the several impacts (Impact 1:strain 0.10, strain rate $11 \mathrm{~s}^{-1}$, Impact 2: 0.15, $21 \mathrm{~s}^{-1}$, Impact 3: 0.22, 27 $\mathrm{s}^{-1}$, Impact 4; 0.30, $38 \mathrm{~s}^{-1}$ ) are expressed as the function of time. The displacements reached at its maximum point in $10 \mathrm{msec}$ by stretching in all four cases and returned to its original position in next $10 \mathrm{msec}$ by releasing (B).

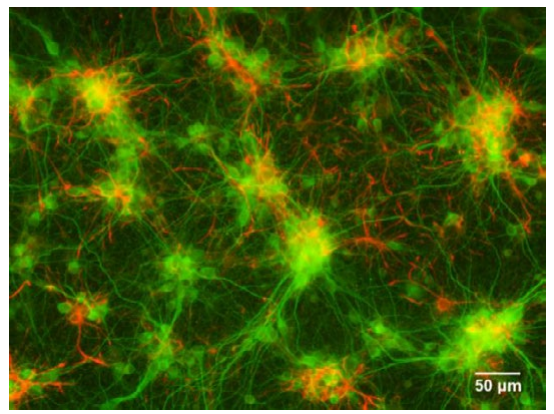

Figure 5. Fluorescent image of cultured neurons

Fluorescence images of primary rat cortex neurons (green) and astrocytes (red). Microtubule-associated protein 2 (MAP2) is stained to label dendrites and glial fibrillary acidic protein (GFAP) is stained to label astrocytes.
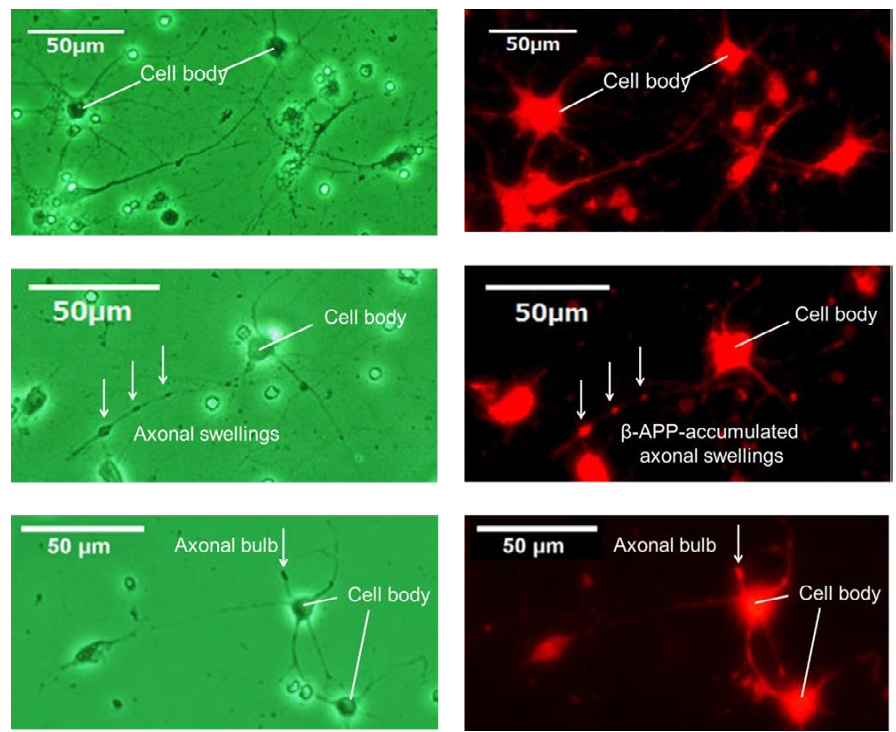

Figure 6. $\beta$-APP immunostaining

Phase-contrast image (left) and fluorescence image (right) of sham control (upper), axonal swellings (middle) and axonal bulb (lower). $\beta$-APP is shown in red. $\beta$-APP accumulated in axonal swellings and axonal bulb by stretching with the strain 0.30 and strain rate $38 \mathrm{~s}^{-1}$.

were analysed totally. Means were compared by Steel's multiple comparison test. A $p$ value of less than 0.05 was considered significant.

\section{Results and discussion}

$\beta$-APP-accumulated axons significantly increased for a strain of 0.22 with a strain rate of $27 \mathrm{~s}^{-1}$ and a strain of 0.30 with a strain rate of $38 \mathrm{~s}^{-1}$ as shown by " $*$ " in Figure 7 in a stretch-dependent manner compared with a sham control. Although the number of axons with swellings significantly increased with increase of strain and strain rate, it can be considered that the generation of the bulb is accompanied with the certain threshold, such as 0.22 and $27 \mathrm{~s}^{-1}$. The rapid stretch of axons can damage the axonal cytoskeleton, resulting in a loss of elasticity and impairment of axoplasmic transport. Subsequent swelling of the axon occurs in discrete bulb formations or in elongated varicosities that accumulate organelles. Transmission electron microscopy (TEM) following in vitro stretch revealed periodic breaks of individual microtubules along axons that regionally corresponded with undulations in axon morphology.

However, typically less than a third of microtubules were broken in any region of an axon. Within hours, these sites of microtubule breaks evolved into periodic swellings, resulting impartial transport interruption [22]. The generation of swellings can be regarded as the temporal dysfunction of the axonal transport and is in the process to secondary axotomy in the disconnection of neurons from target tissues, and cell death.

In summary, the threshold of the dysfunction and the disruption of axonal transport was strain of 0.22 at strain rates of $27 \mathrm{~s}^{-1}$. The electrophysiological threshold of the optic nerve of guinea pig is reported as 0.33 [33], and the threshold of the strain which causes the cell death of the hippocampus of rat brain slice culture is also reported as 0.18 [33]. Both precedent study show that the thresholds for the dysfunction and for the disruption obtained in our study are considerably proper

LaPlaca et al. [24] reported that the strain rate caused cell damage and cell death for 3D cultured neurons was less than $30 \mathrm{~s}^{-1}$. And the undulations can be observed post-injury with at least 0.05 strain [25]. Although more detailed and widely accepted assessment method is required in order to judge the dysfunction of the axonal transport and disconnection of neurons, the observation of the accumulation of $\beta$-APP could still be a one of the most reliable quantitative markers for traumatic axonal injury at a cellular level.

The number of axonal swellings per $100 \mu \mathrm{m}$ increased slightly in
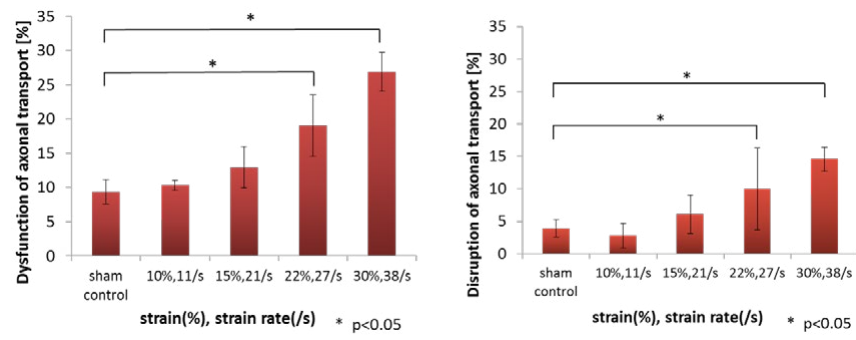

Figure 7. Formation rate of axonal injuries

Rate of neurons that have $\beta$-APP-accumulated axonal swellings (left) and axonal bulbs (right). The four pair of strain and strain rate are also shown in the Figure 4(B). The * symbol represents a statistically significant difference $(p<0.05)$ versus sham control at each condition using Steel's multiple comparison test. Results are expressed as the mean \pm standard deviation (SD) of 4 independent experiments. 
a stretch- dependent manner compared with sham control; however, the changes were not significant among stretching conditions as shown in Figure 8 (left). Similarly, there was no statistical significant change in axonal length of the axon in which axonal bulb was observed as shown in Figure 8 (right). The results mentioned above mean that axonal swellings are formed at the fixed interval regardless of stretching conditions.

Monnerie et al. [26] reported that $70-90 \%$ of beads were separated by $0-10 \mu \mathrm{m}$ regardless of which time-point was examined and that their distribution pattern along the dendrite shaft was similar between $5 \mathrm{~min}$ and $5 \mathrm{~h}$ after axonal stretch injury. Cater et al. [13] also reported that hippocampal cell death is dependent on tissue strain but not strain rate.

Since the recent computational mechanics represented by FEM is developing rapidly and a number of human head FE models have been developed, the tolerances of neurons or the threshold of axonal injury in the mechanical sense is becoming more essential [2729]. Although the Head Injury Criteria (HIC) has been most widely accepted injury criterion, HIC cannot cover the concussive injuries as DAIs and subdural hematomas. Since HIC is only defined by resultant translational acceleration with respect to time, it is difficult to find a correlation between HIC and rotational head motion [30]. The neurons that the axonal direction was controlled were cultured in order to accept a demand in detailed mechanical threshold of the neurons with higher precision. An experimental model that subjected the cultured neurons to uniaxial stretch by controlling the direction of axonal elongation with a microfluidic culture technique was developed by Nakadate $e t$ al. [31]. They showed the difference in the orientation angle of a neurite relative to the tensile direction influenced the formation of swellings, ruptures and retractions of the neurite by using PC12 cells.

On the contrary, concussive mild TBI has been rather spotlighted because despite the name, these injuries are by no means "mild" [25] and the accident itself is sometimes overlooked and repeated. Repeated traumatic injury may result cumulative damage to brain cells and rather serious compared with moderate and severe TBI. Biomechanical study of human concussion that attempts to relate the mechanical input to the localized tissue deformation, such as pressure, stress/strain responses, is needed for a proper assessment of brain injury outcome. There must be a threshold below which no loss of function occurs and a ceiling beyond which irreversible changes in brain function would occur [32]. Post-injury sequelae are difficult to address at the cellular level in vivo. The in vitro studies with experiment was reported by Slemmer et al. [33]. The degrees of membrane deformation result in a biaxial strain or stretch of 0.31 (mild), 0.38 (moderate) and 0.54 (severe), respectively and suggest that the cells of the hippocampus may be susceptible to cumulative damage following repeated mild traumatic insults. Uniaxial stretching device which can control strain and strain
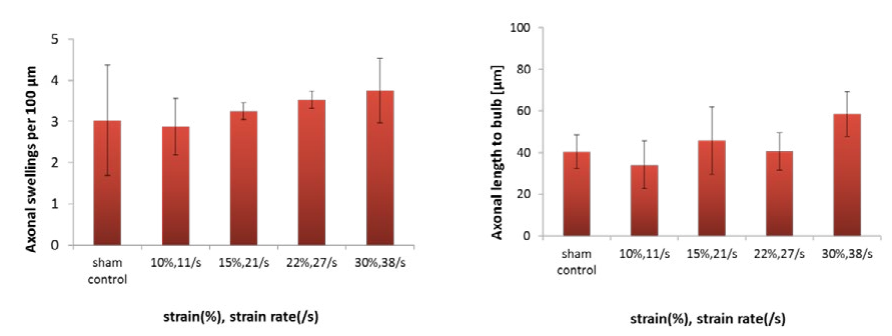

Figure 8. Normalization of axonal injuries

Number of axonal swelling per $100 \mu \mathrm{m}$ (left) and length of axon in which axonal bulb was observed (right). The four pair of strain and strain rate are also shown in the Figure 4(B). rate more independently is expected and will be applied to the neuronal axons those direction are controlled. This may enable to relate the individual neuronal damage to the amount of cumulative damage by repeated insults because the each axon is clearly identified [34].

\section{Conclusions}

The uniaxial stretching device which could give a combination of strain and strain rate to neurons was developed. The axons of the primary rat cortex neurons cultured on a PDMS substrate were stretched by some strains and strain rates. $\beta$-APP was immunostained at $3 \mathrm{~h}$ post loading and observed by fluorescence microscopy. Axonal swellings and bulbs generated on axons after stretching by accumulation of $\beta$-APP were counted by fluorescent images obtained. Dysfunction of the axonal transport was defined as the rate of neurons that have $\beta$-APP-accumulated axonal swellings and disruption of the axonal transport was defined as the rate of neurons that have a $\beta$-APPaccumulated axonal bulb, respectively. The degree of the dysfunction and the disruption of axonal transport progressed with the increase of strain and strain rate. The threshold of dysfunction and disruption of axonal transport was considered as the strain with 0.22 and the strain rate with $27 \mathrm{~s}^{-1}$. The intervals between swellings on an axon are rather constant and are not influenced by the extent of injury nor the magnitude of the strain of the axon.

\section{Competing interests}

The authors declare that they have no competing interests.

\section{Authors' contributions}

SA designed the study. HN developed the device. YK carried out the numerical analysis and observed the immunofluorescence staining. AN participated in the sequence alignment. RW conceived the study, participated in its design and coordination, and helped to draft the manuscript. All authors have read and approved the final manuscript.

\section{Acknowledgements}

This work was partially supported by a Grant-in-Aid for Scientific Research (B) (25289064) and a Grant-in-Aid for Young Scientists (B) (24700459) from the Japan Society for the Promotion of Science.

\section{References}

1. Smith DH, Meaney DF (2000) Axonal Damage in Traumatic Brain Injury. Exp Neurol 6: 483-495.

2. Povlishock JT (1992) Traumatically induced axonal injury: pathogenesis and pathobiological implications. Brain Pathol 2: 1-12. [Crossref]

3. McIntosh TK, Smith DH, Meaney DF, Kotapka MJ, Gennarelli TA, et al. (1996) Neuropathological sequelae of traumatic brain injury: relationship to neurochemical and biomechanical mechanisms. Lab Invest 74: 315-342. [Crossref]

4. Gaetz M (2004) The neurophysiology of brain injury. Clin Neurophysiol 115: 4-18 [Crossref]

5. Povlishock JT, Christman CW (1995) The pathobiology of traumatically induced axonal injury in animals and humans: a review of current thoughts. J Neurotrauma 12 : 555-564. [Crossref]

6. Pilz P (1983) Axonal injury in head injury. Acta Neurochir Suppl (Wien) 32: 119-123.

7. Koo EH, Sisodia SS, Archer DR, Martin LJ, Weidemann A, et al. (1990) Precursor of amyloid protein in Alzheimer disease undergoes fast anterograde axonal transport. Proc Natl Acad Sci U S A 87: 1561-1565. [Crossref]

8. Gentleman SM, Nash MJ, Sweeting CJ, Graham DI, Roberts GW (1993) Beta- amyloid precursor protein (beta APP) as a marker for axonal injury after head injury. Neurosci Lett 160: 139-144. [Crossref]

9. Blumbergs PC, Scott G, Manavis J, Wainwright H, Simpson DA, et al. (1995) 
Topography of axonal injury as defined by amyloid precursor protein and the sector scoring method in mild and severe closed head injury. $J$ Neurotrauma 12: 565-572. [Crossref]

10. McKenzie KJ, McLellan DR, Gentleman SM, Maxwell WL, Gennarelli TA, et al. (1996) Is beta-APP a marker of axonal damage in short-surviving head injury? Acta Neuropathol 92: 608-613. [Crossref]

11. Povlishock JT, Jenkins LW (1995) Are the pathobiological changes evoked by traumatic brain injury immediate and irreversible?. Brain Pathol 5: 415-426. [Crossref]

12. Wilkinson AE, Bridges LR, Sivaloganathan S (1999) Correlation of survival time with size of axonal swellings in diffuse axonal injury. Acta Neuropathol 98: 197-202. [Crossref]

13. Cater HL, Sundstrom LE, Morrison B $3^{\text {rd }}$ (2006) Temporal development of hippocampal cell death is dependent on tissue strain but not strain rate. J Biomech 39: 2810-2818. [Crossref]

14. Pfister BJ, Weihs TP, Betenbaugh M, Bao G (2003) An in vitro uniaxial stretch mode for axonal injury. Ann Biomed Eng 31: 589-598. [Crossref]

15. Kilinc D, Gallo, G, Barbee KA (2008) Mechanically-induced membrane poration causes axonal beading and localized cytoskeletal damage. Exp Neurol 212: 422-230. [Crossref]

16. Kilinc D, Gallo G, Barbee KA (2009) Mechanical membrane injury induces axonal beading through localized activation of calpain. Exp Neurol 219: 553-561. [Crossref]

17. Smith DH, Wolf JA, Lusardi TA, Lee VM, Meaney DF (1999) High tolerance and delayed elastic response of cultured axons to dynamic stretch injury. $J$ Neurosci 19 : 4263-4269. [Crossref]

18. Armani D, Liu C, Aluru N (1999) Re-configurable fluid circuits by PDMS elastomer micromachining. Proc IEEE MEMS: 222-227.

19. Rand CW, Courville CB (1946) Histologic changes in the brain in cases of fatal injury to the head; alterations in nerve cells. Arch Neurol Psychiatry 55: 79-110. [Crossref]

20. Smith DH, Meaney DF, Shull WH (2003) Diffuse axonal injury in head trauma. J Head Trauma Rehabil 18: 307-316. [Crossref]

21. Strich SJ (1956) Diffuse degeneration of the cerebral white matter in severe dementia following head injury. J Neurol Neurosurg Psychiatry 19: 163-185. [Crossref]

22. Tang-Schomer MD, Johnson VE, Baas PW, Stewart W, Smith DH (2012) Partial interruption of axonal transport due tomicrotubule breakage accounts for the formation of periodic varicosities after traumatic axonal injury. Exp Neurol 233: 364-372. [Crossref]

23. Bain AC, Meaney DF (2000) Tissue-level thresholds for axonal damage in an experimental model of central nervous system white matter injury. J Biomech Eng 122: 615- 622. [Crossref]

24. LaPlaca MC, Cullen DK, McLouchlin JJ, Cargill RS (2005) High rate shear strain of three-dimensional neural cell cultures: a new in vitro traumatic brain injury model. $J$ Biomech 38: 1093-1105. [Crossref]

25. Yuen TJ, Browne KD, Iwata A, Smith DH (2009) Sodium channelopathy induced by mild axonal trauma worsens outcome after a repeat injury. $J$ Neurosci Res 87: 3620 3625. [Crossref]

26. Monnerie H, Tang-Schomer MD, Iwata A, Smith DH, Kim HA, et al. (2010) Dendritic alterations after dynamic axonal stretch injury in vitro. Exp Neurol 224: 415-423. [Crossref]

27. LaPlaca MC, Prado GR (2010) Neural mechanobiology and neuronal vulnerability to traumatic loading. J Biomech 43: 71-78. [Crossref]

28. Willinger R, Baumgartner D (2003) Human head tolerance limits to specific injury mechanics. Int J Crash 8: 605-617.

29. Deck C, Willinger R (2008) Improved head injury criteria based on head FE model. Int J Crash 13: 667-678.

30. Kimpara H, Iwamoto M (2012) Mild traumatic brain injury predictors based on angular acceleration during impacts. Ann Biomed Eng 40: 114-126. [Crossref]

31. Nakadate H, Fukumura Y, Kaneko Y, Kakuta A, Furukawa H, et al. (2014) In vitro uniaxial stretch model for evaluating the effect of strain along axon on damage to neurons. J Biomech Sci Eng 9: 14-00136.

32. Zhang L, Yang KH, King AI (2004) A proposed injury threshold for mild traumatic brain injury. J Biomech Eng 126: 226-236. [Crossref]

33. Slemmer JE, Master EJT, Zeeuw CIDe, Weber JT (2002) Repeated mild injury cause cumulative damage to hippocampal cells. Brain 125: 2699-2709. [Crossref]

34. Morrison III B, Cater HL, Wang CB, Thomas FC, Hung CT, et al. (2003) A tissue level tolerance criteria for living brain developed with an in vitro model of traumatic mechanical loading. Stapp Car Crash J 47: 93-105. [Crossref]

Copyright: (C2016 Aomura S. This is an open-access article distributed under the terms of the Creative Commons Attribution License, which permits unrestricted use, distribution, and reproduction in any medium, provided the original author and source are credited. 